\title{
Erythrocyte Sequestration and Anemia in Severe Falciparum Malaria Analysis of Acute Changes in Venous Hematocrit Using a Simple Mathematical Model
}

Timothy M. E. Davis, Sanjeev Krishna, Sornchai Looareesuwan, Wichai Supanaranond, Sasithon Pukrittayakamee, Kunya Attatamsoonthom, and Nicholas J. White

Faculty of Tropical Medicine, Mahidol University, Bangkok 10400, Thailand; Tropical Medicine Unit, Nuffield Department of Clinical Medicine, John Radcliffe Hospital, Oxford OX3 9DU, England; and Intensive Care Unit, Paholpolpayuhasena Hospital, Kanchanaburi, Thailand

\begin{abstract}
Microvascular erythrocyte sequestration, the characteristic pathological feature of falciparum malaria, was evaluated using a mathematical model in $\mathbf{4 6}$ patients with severe infections. From admission radioisotopic circulating red cell volumes and simultaneous venous hematocrits, the model-derived sequestrum hematocrit (mean [95\% confidence limits]: 0.70 $[0.43-0.97], n=29)$ was twice that of peripheral blood $(0.33$ $[0.30-0.36])$. Serial reticulocyte and radiolabeled erythrocyte counts indicated that small numbers of cells enter the circulation during initial therapy. The mean fall in hematocrit over $84 \mathrm{~h}$ in 26 nontransfused patients conformed to a three-term equation. A first-order decline $\left(t_{1 / 2} 2.0 \mathrm{~h}[0.6-3.4]\right)$ suggested an average $7.5 \%$ plasma volume expansion through rehydration. A zero-order 6.3\% (3.1-9.5) fall ( $t_{1 / 2} 25.7$ h [21.2-30.2]) occurred contemporaneously with a fall in mean parasitemia from $4.5 \%$ (3.6-5.4); from these data the model-derived average sequestered erythrocyte volume $(4.8 \%$ of the admission hematocrit) was similar to the peripheral parasite burden. $A$ second, first-order fall $\left(t_{1 / 2} 1,047 \mathrm{~h}[278-1,816]\right)$ indicated loss of uninfected erythrocytes with mean lifespan $62 \mathrm{~d}$. Predicted total plasma volume expansion during initial therapy (21.2\%) was similar to radioisotopic estimates in 11 patients $(17.3 \%$ [2.0-33.1]). Application of the model to individual patient data showed wide variations in relative proportions of circulating and sequestered parasitized cells. The model provides evidence of the nature and fate of all parasitized erythrocytes in malaria. (J. Clin. Invest. 1990. 86:793-800.) Key words: anemia • falciparum malaria $\cdot$ sequestration
\end{abstract}

\section{Introduction}

The characteristic pathological feature of falciparum malaria is the adherence of erythrocytes containing mature forms of the parasite to venous and capillary endothelium in organs such as brain, heart, and kidney (1). Such deep vascular "sequestration" is thought to be a major factor in the genesis of vital organ dysfunction (2) and may also contribute to the rapid

Address reprint requests to Dr. Timothy M. E. Davis, Faculty of Tropical Medicine, Mahidol University, 420/6 Rajvithi Road, Bangkok 10400, Thailand.

Received for publication 15 November 1988 and in revised form 5 February 1990

J. Clin. Invest.

(C) The American Society for Clinical Investigation, Inc.

0021-9738/90/09/0793/08 $\$ 2.00$

Volume 86, September 1990, 793-800 development of anemia in severe infections (3). Quantitation of the hidden mass of pathogenic, sequestered, parasitized cells has so far eluded estimation in vivo. As a consequence, the clinician managing a patient with severe malaria cannot be certain of the total parasite burden, a factor that may have considerable bearing on management and prognosis. A pertinent example of this dilemma is the assessment of a rapidly falling hematocrit against a background of delicate fluid balance and the practical need to predict transfusion requirements well in advance. To understand serial changes in hematocrit and provide an estimate of erythrocyte sequestration in patients with severe falciparum malaria, we have used a simple, two-compartment model to examine the relationship between circulating and sequestered red cell volumes before and during initial therapy.

\section{Methods}

\section{The model}

A simple model of intravascular blood distribution in falciparum malaria is one in which circulating red cells and plasma form one compartment and sequestered erythrocytes and plasma another (Fig. 1). Although the relative volume of sequestered plasma will be assumed obligatory and hence the sequestrum hematocrit, $s$, a constant, plasma can circulate between compartments. By contrast, sequestered erythrocytes will be considered unable to reenter the circulation (consistent with the observation that mature trophozoites and schizonts are seldom seen in peripheral blood smears), and the numbers of parasitized cells that sequester after treatment has started will be taken to be small. It will also be assumed that the venous hematocrit bears a constant relationship to the total circulating packed cell volume during initial treatment.

In an individual with total body hematocrit, $h_{0}$, and red cell volume, $r_{0}$, at $100 \%$ of that predicted for age and sex, the relationship between $h_{0}$ and $r_{0}$ is given by

$$
h_{0}=\frac{r_{0}}{r_{0}+p_{0}},
$$

where $p_{0}$ is the circulating plasma volume. With $p_{0}$ expressed as a percentage relative to $r_{0}=100$, Eq. 1 can be reexpressed as

$$
p_{0}=\frac{100}{h_{0}}-100 \text {. }
$$

In the presence of sequestered cells, the peripheral venous hematocrit $h$ will have the value

$$
h=\frac{r}{r+p},
$$

where $r$ is the circulating red cell volume (exclusive of sequestered cells), $p$ is the corresponding circulating plasma volume, and both $r$ and $p$ have values relative to $r_{0}=100$. Since $100-r$ represents the 


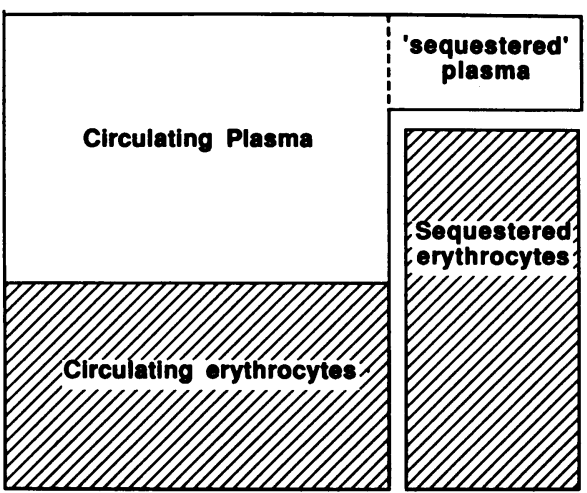

Total Blood Volume

Figure 1. Diagrammatic representation of the division of red cells and plasma into two compartments in falciparum malaria. The total intravascular volume consists of circulating and sequestered erythrocytes, and freely circulating plasma. Sequestered erythrocytes remain fixed and unable to reenter the circulation and their associated hematocrit, $s$ is constant.

simultaneous sequestered cell volume, $s$ can be expressed in the form of Eq. 3 above as

$$
s=\frac{100-r}{(100-r)+\left(p_{0}-p\right)} .
$$

Using this equation, and Eqs. 2 and 3 above, the relationship between venous hematocrit and circulating red cell volume can be reexpressed as

$$
h=\frac{r h_{0} s}{100 s-h_{0}(100-r)} .
$$

The effect of different values of $s$ on this relationship is shown in Fig. 2. As increased volumes of plasma are obligatorily sequestered, the value of $s$ will decrease and the curve will shift to the left (see Fig. 2). As the value of $s$ approaches that of $h_{0}$, the curve tends to the horizontal.

Changes in circulating red cell and plasma volumes. So far, the model describes changes in relative amounts of erythrocytes and

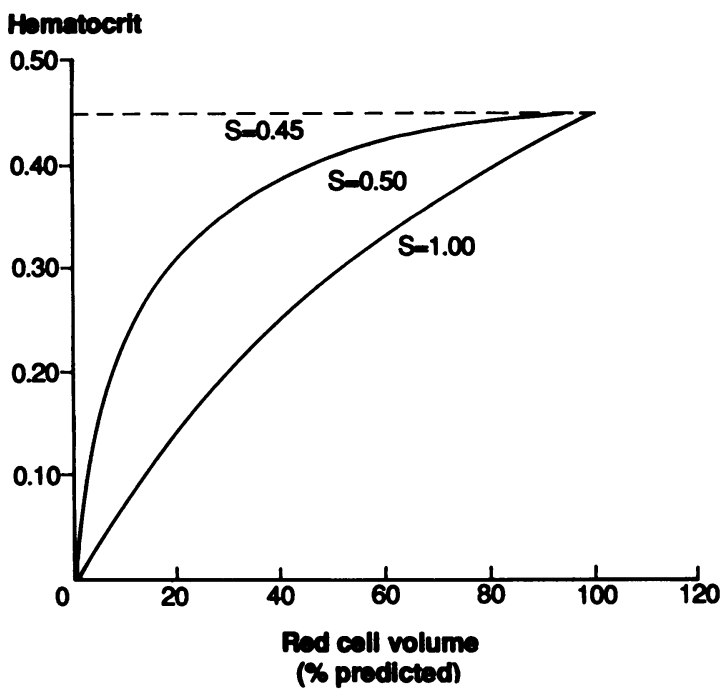

Figure 2. Relationship between peripheral venous hematocrit and circulating red cell volume for sequestration occurring at values of

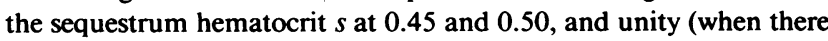
is no sequestered plasma). plasma within a total intravascular volume which remains $r_{0}+p_{0}$. The loss of circulating red cells or plasma through processes other than sequestration will affect the relationship between $h$ and $r$ shown in Fig. 2 but will not influence the value of $s$. Loss of plasma (hemoconcentration) would increase $h_{0}$ at $r_{0}=100$ according to the equation:

$$
h_{0}{ }^{1}=\frac{r_{0}}{r_{0}+\left(p_{0}-x p_{0}\right)}=\frac{h_{0}}{x\left(h_{0}-1\right)+1},
$$

where $x$ is the proportion of plasma lost and $h_{0}{ }^{1}$ the new "initial" hematocrit at $r_{0}=100$. Substitution of $h_{0}$ for $h$ in Eq. 4 describes the relationship between $h$ and $r$ for sequestration in the presence of a known amount of plasma depletion, shifting the original curve to the left (see Fig. 3). Red cell loss (relative hemodilution) would decrease the value of $h_{0}$ according to the relationship

$$
h_{0}^{2}=\frac{r_{0}-x r_{0}}{\left(r_{0}-x r_{0}\right)+p_{0}}=\frac{h_{0}-x h_{0}}{1-x h_{0}},
$$

where $x$ was the proportion of red cells lost and $h_{0}^{2}$ the new initial hematocrit at $r_{0}^{2}=100(1-x)$. Substitution of $h_{0}^{2}$ for $h_{0}$ and $r_{0}^{2}$ for 100 in Eq. 4 describes the relationship between $h$ and $r$ for sequestration in the presence of red cell destruction, shifting the curve to the right (see Fig. 3). Substitution of $100(1-x)$ for $r$ and 1.0 for $s$ in Eq. 4 yields Eq. 6 above, indicating that pure red cell loss has a nonlinear relationship with venous hematocrit identical to that for sequestration at $s=1.0$.

Changes in the venous hematocrit due to rehydration and antimalarial therapy. During management of acute malaria, changes in the peripheral venous hematocrit reflect changes in the relative amounts of circulating erythrocytes and plasma within the two-compartment intravascular space. Under the model's premises, parasitized red cells do not pass between compartments and no new erythrocytes enter the circulation. It will also be assumed that changes in plasma volume occur in two stages: a rapid initial increase reflecting restoration of an appropriate circulating blood volume, followed by a slower progressive expansion to maintain this blood volume in the presence of accelerated erythrocyte destruction. The fall in hematocrit during initial rehydration, which is independent of simultaneous red cell loss, can be repre-

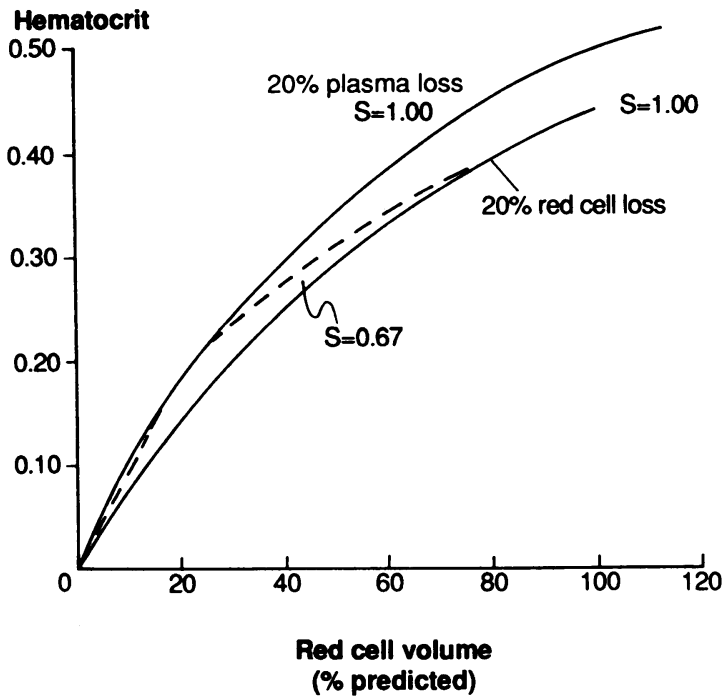

Figure 3. Relationship between peripheral venous hematocrit and circulating red cell volume for sequestration with $(a)$ an associated hematocrit $s$ of 1.0 (with no associated plasma) in the presence of $20 \%$ plasma loss (shifting the curve shown in Fig. 2 to the left), and (b) an $s$ of 0.67 after a $20 \%$ loss of red cells from the circulation (dashed line). The latter line would be to the right of that with $s$ of 0.67 and no prior red cell loss. 
sented as a function $(f)$ of time $(t)$ after initiation of treatment such that $h=f_{1}(t)$.

Sequestered erythrocytes will be taken to be composed entirely of cytoadherent parasitized cells with similar clearance kinetics to those of circulating parasitized erythrocytes. The "space" created by destruction of both parasitized cell populations will be filled by the second phase of compensatory plasma volume expansion and the fall in hematocrit will be a second function of time: $h=f_{2}(t)$. Clearance of uninfected erythrocytes will also be matched by plasma volume expansion. The resulting change in hematocrit will be a third function of time: $h=f_{3}(t)$.

The combined effects of rehydration and clearance of nonparasitized and sequestered and circulating parasitized cells will be represented by the function:

$$
h=f_{1}(t)+f_{2}(t)+f_{3}(t),
$$

where $f_{1}, f_{2}$, and $f_{3}$ are contemporaneous zero- or first-order kinetic processes of the form: $A-k \cdot t$ or $A \cdot e^{-k \cdot t}$, where $A$ and $k$ are constants.

Under the model's assumptions and Eq. 7 above, the coefficient $A$ in $f_{1}(t)$ represents initial plasma volume expansion without coincidental red cell loss. Eq. 5 above can be rewritten as

$$
h_{1}=\frac{r}{r+p+v \cdot p},
$$

where $h_{1}$ is venous hematocrit predicted after a fall of $A$ from its initial value $h_{0}$, and $v$ is the proportion of the circulating plasma volume $p$ replaced during rehydration. Amalgamation of Eq. 3 and 8 above yields the equation:

$$
v=\frac{\left(h_{0}-h_{1}\right)}{h_{1}\left(1-h_{1}\right)} .
$$

The second component of the hematocrit-time curve $\left[f_{2}(t)\right]$ reflects destruction of all parasitized cells. The plasma volume will expand in compensation and the venous hematocrit will become an amalgamation of Eqs. 5 and 6 above:

$$
h_{2}=\frac{r-i}{(r-i)+(p+i)+v+q},
$$

where $h_{2}$ is the hematocrit after a further fall from $h_{1}, i$ the volume of circulating, infected cells, and $q$ the total sequestrum volume. Using Eqs. 3 and 9 above, Eq. 10 simplifies to

$$
q=\left[\frac{(1-i)}{h_{2}}-\frac{1}{h_{1}}\right] \cdot r .
$$

This relationship provides the total sequestrum volume in terms of the circulating red cell volume $r$. Therefore, if a valid estimate of $s$ is available, the model allows estimation of the sequestered cell volume at presentation through interpretation of subsequent changes in the peripheral venous hematocrit. Other indirect estimates are also provided: the rate and volume of initial rehydration, the underlying rate of loss of uninfected erythrocytes, and the total plasma volume increase during initial therapy.

\section{Validation and application of the model}

To assess the validity of the model's assumptions, four studies were performed in adult patients with severe falciparum malaria:

1. The measurement of circulating red cell volume and peripheral venous hematocrit to determine whether Eq. 4 above adequately describes the relationship between these two variables.

2. The serial measurement of the proportion of radiochromiumlabeled erythrocytes and reticulocytes in nontransfused patients to determine whether significant numbers of red cells enter the circulation from either the bone marrow or sequestered cell mass after the initiation of treatment.

3. The serial measurement of venous hematocrit and peripheral parasitemia in nontransfused patients to determine whether the se- quential fall in hematocrit attributable to rehydration and destruction of parasitized (circulating and sequestered) and nonparasitized erythrocytes is adequately described by the model.

4. The radioisotopic measurement of plasma volume in nontransfused patients before and after initial therapy to determine whether actual increases in plasma volume are similar to those predicted by the model from the fall in venous hematocrit.

Patients. Two groups of patients were studied (Table I). For studies 1-3 above, 35 patients were recruited (group 1). Study 4 involved a further 11 patients (group 2). All presented to Paholpolpayuhasena Hospital, Kanchanaburi, Thailand between May 1987 and June 1989. Each patient had asexual forms of Plasmodium falciparum on peripheral blood smear and was assessed as having severe disease with at least one of the following features (4): coma, jaundice, anemia, dehydration and oliguria, hypoglycemia, or hyperparasitemia (parasite count $>250,000 / \mu \mathrm{l})$. Women of child-bearing age and children under $11 \mathrm{yr}$ of age were excluded. Fully informed consent was obtained from the patient or an attendant relative before study entry. The study was approved by the Ethics Committee of the Faculty of Tropical Medicine, Mahidol University, Bangkok, Thailand.

Clinical management. All patients were started on intravenous fluids on admission. After confirmation of the diagnosis by blood smear examination, quinine was given by intravenous infusions (5-7). Fluid administration was monitored by regular measurement of the central venous pressure or assessment of the jugular venous pulse so as to maintain a central venous pressure of $<5 \mathrm{~cm}$ water. Patients with venous hematocrits below $21 \%$ received a transfusion of at least two units of packed red cells. Neurological, renal, respiratory, and cardiovascular complications were treated as described previously (4). Intravenous therapy was continued until a satisfactory oral intake was established and antimalarial drugs could be given by mouth

Radiochromium labeling and determination of red cell volumes. Radioisotopic measurement of the circulating red cell volume was performed as soon as possible after admission, as quinine was being given and, in severely anemic patients, before transfusion. Radiolabeling of autologous erythrocytes was as described previously $(8,9)$. A $10-\mathrm{ml} \mathrm{blood} \mathrm{sample} \mathrm{was} \mathrm{collected} \mathrm{into} 2 \mathrm{ml}$ of acid-citrate-dextrose anticoagulant and $50 \mu \mathrm{Ci}$ of sodium chromate $\left({ }^{51} \mathrm{Cr}\right.$ ) solution (Amersham International, Amersham, England) was added dropwise with careful mixing over $30 \mathrm{~min}$ followed by a solution of $25 \mathrm{mg}$ of ascorbic acid. A 2-ml aliquot of the labeled sample was retained and the remainder was injected intravenously back into the patient. After 10 min, a 10-ml sample was taken from the opposite arm. Half this sample was centrifuged and the separated plasma and the other $5 \mathrm{ml}$ of

\begin{tabular}{|c|c|c|c|c|}
\hline & \multicolumn{2}{|r|}{$\begin{array}{l}\text { Group } 1 \\
(n=35) \\
\end{array}$} & \multicolumn{2}{|r|}{$\begin{array}{l}\text { Group } 2 \\
(n=11)\end{array}$} \\
\hline & Mean & Range & Mean & Range \\
\hline Age $(y r)$ & 26.4 & $(11-57)$ & 35.2 & $(15-69)$ \\
\hline Sex (males/females) & $24 / 11$ & & $9 / 2$ & \\
\hline Oral temperature & & & & \\
\hline$\left({ }^{\circ} \mathrm{C}\right)$ & 38.6 & $(36.3-40.5)$ & 37.6 & $(36.0-39.2)$ \\
\hline Venous hematocrit & 0.33 & $(0.15-0.48)$ & 0.36 & $(0.25-0.44)$ \\
\hline Parasite count & & & & \\
\hline$($ per $\mu l)$ & $79,809^{*}$ & $(108-869,152)$ & $72,984^{*}$ & $(270-519,230)$ \\
\hline $\begin{array}{l}\text { Serum creatinine } \\
\qquad(m g / d l)\end{array}$ & 1.4 & $(0.4-5.7)$ & 2.2 & $(1.1-5.4)$ \\
\hline $\begin{array}{l}\text { Serum total bilirubin } \\
(m g / d l)\end{array}$ & 3.6 & $(0.2-19.5)$ & 4.7 & $(0.2-22.0)$ \\
\hline
\end{tabular}
whole blood were retained for counting. Red cell volume was calculated from whole-blood counts and hematocrits of both the injectate

Table I. Admission Clinical and Laboratory Data from 46 Patients with Severe Malaria

* Geometric mean. 
and 10-min sample after correction for plasma radioactivity (10). Patients in group 1 constituted a heterogeneous sample in terms of age and sex distribution (Table I), two important influences on body weight-corrected red cell mass (11). To allow valid comparisons between patients, each red cell volume in milliliters per kilogram of body weight was converted to a percentage of that predicted for age and $\operatorname{sex}(11)$.

During the first $\mathbf{4} \mathrm{d}$ of hospitalization, further blood samples were taken at least twice daily for counting and measurement of the venous microhematocrit and parasite count. As the proportion of parasitized erythrocytes in the labeled sample was small, the effect of alterations in ${ }^{51} \mathrm{Cr}$ uptake by, and elution from, infected cells on whole-blood radioactivity was taken as minimal. It was also assumed that the number of labeled, parasitized cells continuing to sequester despite quinine treatment was also small. Therefore, all counts were corrected for ${ }^{51} \mathrm{Cr}$ decay and $1.0 \% / \mathrm{d}$ elution (12), and expressed as a percentage of the corrected counts of the 10-min sample.

Determination of plasma volumes. Measurement of plasma volumes was as described previously (13). A 5- $\mathrm{Ci}$ aliquot of an aqueous solution of ${ }^{125}$ I-labeled human albumin (Amersham International) was added to $10 \mathrm{ml}$ of sterile isotonic saline solution and $0.5 \mathrm{ml}$ of the mixture was retained. The remainder was injected into the patient as an intravenous bolus at $0 \mathrm{~min}$. Blood samples were drawn from the opposite arm at 10,15, 20,30,45, and $60 \mathrm{~min}$. After centrifugation, the radioactivity of each plasma sample was determined and the plasma volume was calculated from the radioactivity of the injectate, the volume injected, and linear extrapolation of log-transformed plasma counts to $0 \mathrm{~min}$. All patients in group 2 had a plasma volume determination on admission and a second after 3-4 d of treatment.

Statistical analysis and curve fitting. Statistical analysis was by parametric tests (14) and data are, unless otherwise indicated, expressed as means and SEM. The iterative nonlinear curve-fitting program PCNONLIN (15) was used to fit Eqs. 4 and 7 above to raw data. Where mean values were submitted to PCNONLIN, the reciprocal of the SEM was used to weight data. The adequacy of fit was gauged from the magnitude of the weighted sum of squares of residuals, the presence of patterns among the residuals, and the correlation between observed and fitted values of the independent variable. Comparison of a constant $(c)$ derived from regression analysis of $n$ values to a theoretical value $\left(c_{t}\right)$ was through the use of the formula: $t=\left(c-c_{t}\right) /[\mathrm{SE}(c)]$ with $n$ -2 degrees of freedom $(d f)$.

Serial hematocrit data from each patient were expressed as a percentage of the admission value to minimize the effect on the absolute mean of values missing because some patients with low hematocrits were transfused after $48 \mathrm{~h}$ of treatment. Missing single data points lying between two valid hematocrits were taken as the geometric mean of adjacent values, but there was no extrapolation beyond the final hematocrit of each patient. Parasite counts on simultaneous blood smears were expressed per 1,000 erythrocytes and then as a percentage of the admission value.

\section{Results}

Clinical course. Of the 46 patients studied, 43 survived and were discharged from hospital. Three patients in group 1 , all in unrousable coma, died within $4 \mathrm{~d}$ of admission. 12 group 1 patients were transfused during the period of study. Parasite clearance, taken as the first of two consecutive parasite-negative thick blood films, took an average of $64 \mathrm{~h}$ (absolute range 8-119) in 38 assessable patients. None of the surviving patients developed evidence of recrudescent infection, hemorrhage, or sepsis within $84 \mathrm{~h}$ of admission.

Red cell volume and its relationship to venous hematocrit (study 1). The admission circulating red cell volumes and simultaneous uncorrected venous hematocrits of 29 patients were shown in Fig. 4. Eq. 4 was fitted to the data and estimates

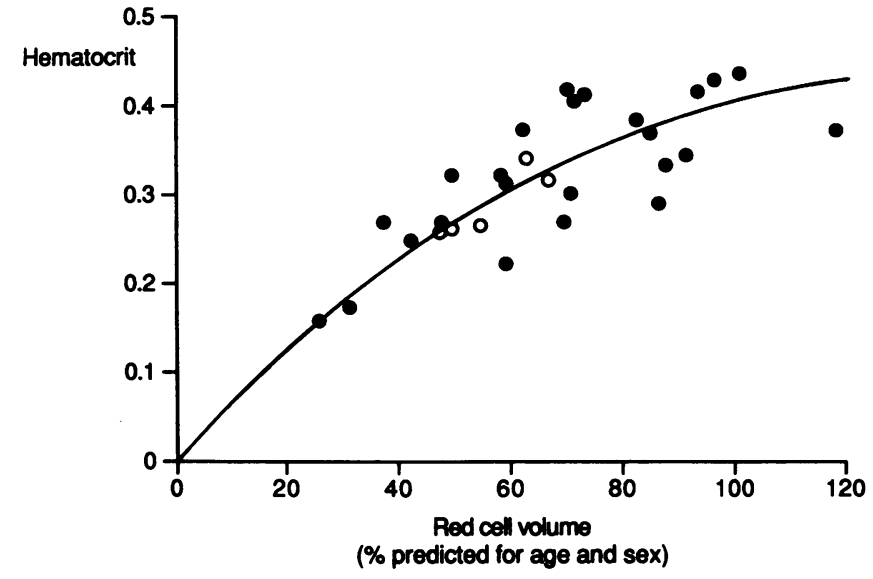

Figure 4. Relationship between circulating red cell volume as a percentage of that predicted for age and sex, and simultaneous uncorrected peripheral venous hematocrit for 29 patients with severe falciparum malaria. (0) Patients with cerebral malaria. The solid line is the curve representing Eq. 4 fitted to the data with a resultant mean estimate of $s$ of 0.70 .

$( \pm \mathrm{SE})$ for $h_{0}(0.39 \pm 0.02 ; 95 \%$ confidence limits $0.36-0.42)$ and $s(0.700 \pm 0.130 ; 0.43-0.97)$ were obtained. The value of $s$ was significantly different from $1.00(t=2.308, d f=27, P$ $=0.029$ ). The coordinates of the five patients with cerebral malaria were all close to the fitted curve.

Serial changes in the proportion of radiochromium-labeled erythrocytes and in the reticulocyte count (study 2). Serial radiochromium data were available for 27 nontransfused patients at least four time points during a minimum of $48 \mathrm{~h}$ after admission. The slope of the regression line relating corrected counts per milliliter of red cells as a percentage of the 10-min value to the time after admission was not significantly different from zero in 19 patients $(-1.81 \leq t \leq 1.88,2 \leq d f \leq 6, P$ $>0.05$ ). In six patients, the slope was significantly less than zero $(t \leq-2.15, d f \geq 4, P<0.05)$ and in two the slope was significantly greater than zero $(t \geq 2.11, d f \geq 5, P<0.05)$. The mean \pm SEM intercepts and slopes of the 27 regression lines were $98.5 \pm 2.4 \%$ and $-0.022 \pm 0.052$, respectively, and the mean extrapolated value at $84 \mathrm{~h}(96.8 \pm 3.6 \%)$ was not significantly different from the corresponding intercept $(t=0.43, d f$ $=26, P>0.6$ ).

The median daily reticulocyte count in a subset of eight patients remained at or below $0.2 \%$ during the first $4 \mathrm{~d}$ of treatment (Table II).

Serial changes in the venous hematocrit and parasite count during the first $84 \mathrm{~h}$ of admission (study 3). To first determine and validate the kinetic processes that best describe serial

Table II. Serial Reticulocyte Counts from Eight Patients with Severe Falciparum Malaria

\begin{tabular}{lcccc}
\hline & \multicolumn{5}{c}{ Days after admission } \\
\cline { 2 - 5 } & 0 & 1 & 2 & 3 \\
\hline Median (\%) & 0.1 & 0.1 & 0.2 & 0.2 \\
Range (\%) & $<0.1-0.8$ & $<0.1-5.1$ & $<0.1-7.6$ & $<0.1-4.1$
\end{tabular}


changes in the venous hematocrit in patients with severe malaria, pooled data from suitable group 1 patients were used. This approach made maximal use of available hematocrittime data and minimized the effects of errors in individual hematocrit values when different equations with multiple parameters were compared over limited numbers of coordinates. At least four serial venous hematocrits over at least $48 \mathrm{~h}$ were available for each of 26 nontransfused patients. An average of 2.4 values at each of the eight time points were determined by logarithmic interpolation (range 0-6). Eq. 7 above was fitted to mean data (Fig. 5) and the combination of terms that produced the best fit was one in which $f_{1}(t)$ and $f_{3}(t)$ were first order and $f_{2}(t)$ was zero order: i.e.,

$$
h=A e^{-a \cdot t}+(B-b \cdot t)+C e^{-c \cdot t},
$$

where fitted values $\pm \mathrm{SE}$ for $A, B, C, a, b$, and $c$ are shown in Table III.

As assumed by the model, the middle term in Eq. $12\left[f_{2}(t)\right]$ is a zero-order fall representing destruction of all parasitized cells. To determine whether the peripheral clearance of parasitized cells also approximates to a zero-order process, serial parasite counts were analyzed. The mean admission parasitemia of 18 group 1 patients with at least three thin-film parasite counts between 0 and $36 \mathrm{~h}$ was 56 per 1,000 red cells $(5.6 \%$; absolute range $2.1-14.3$ ). Serial parasite counts from each patient were expressed as a percentage of baseline. To limit the influence of errors in parasite counts on percentage changes in patients with low admission parasitemias, geometric means were calculated at each time point (Fig. 6). Linear regression analysis of geometric mean values $(y)$ at five time points between 0 and $36 \mathrm{~h}$ yielded the equation: $y=97.0-2.792 t$, where $t$ is the time after admission $(r=-0.97, n=5, P$ $<0.005)$.

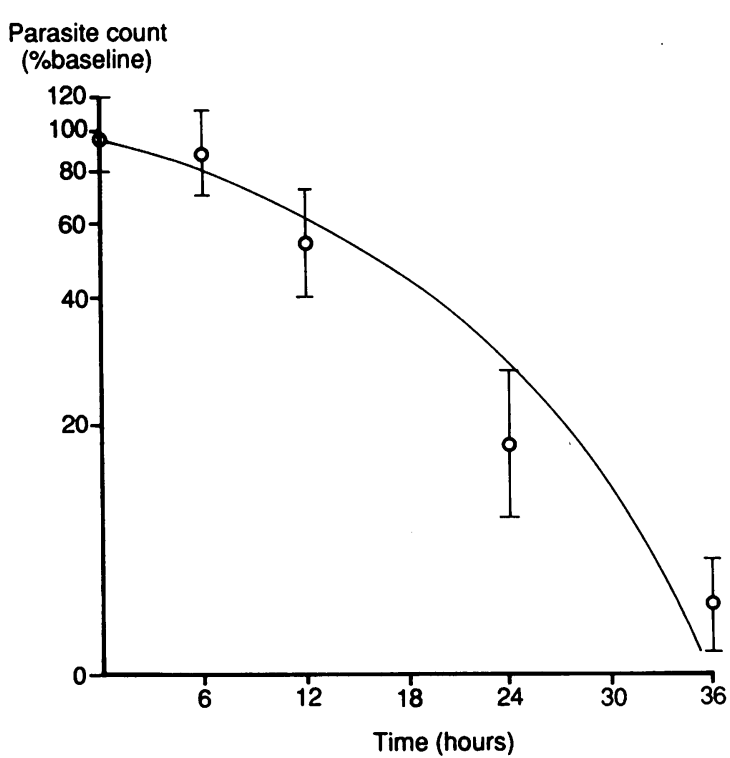

Figure 5. Semilogarithmic plot of mean \pm SEM of venous hematocrits expressed as a percentage of the admission value (baseline) for $26 \mathrm{pa}-$ tients with severe falciparum malaria. Numbers of data at each time point are shown in parentheses. The solid line represents Eq. 12 fitted to the data.
Table III. Estimated Values $\pm S E$ of Parameters Derived from Eq. 12 Fitted to Mean Data from 26 Nontransfused Patients (see Fig. 5) and to Serial Venous Hematocrit Values from 18 Individual Group 1 Patients (Mean \pm SEM)

\begin{tabular}{lcc}
\hline & $\begin{array}{c}\text { Mean data } \\
(n=26)\end{array}$ & $\begin{array}{c}\text { Individual cases } \\
(n=18)\end{array}$ \\
\hline$A(\%)$ & $4.8 \pm 0.5$ & $3.9 \pm 1.7$ \\
$a\left(h^{-l}\right)$ & $0.34 \pm 0.11$ & $0.47 \pm 0.26$ \\
$t_{1 / 2}(h)$ & $2.0 \pm 0.7$ & $1.5 \pm 1.8$ \\
$B(\%)$ & $6.3 \pm 1.6$ & $10.3 \pm 1.8$ \\
$b\left(\% \cdot h^{-1}\right)$ & $0.12 \pm 0.02$ & $0.34 \pm 0.08$ \\
$t_{1 / 2}(h)$ & $25.7 \pm 2.2$ & $24.9 \pm 3.6$ \\
$C(\%)$ & $89.0 \pm 1.5$ & $86.8 \pm 2.1$ \\
$c\left(h^{-l}\right)$ & $0.00066 \pm 0.00023$ & $0.00096 \pm 0.00336$ \\
$t_{1 / 2}(h)$ & $1047 \pm 374$ & $722 \pm 560$ \\
\end{tabular}

Mean \pm SE of half-times $\left(t_{1 / 2}\right)$ calculated from the rate constants $a, b$, and $c$ are also shown.

Changes in plasma volume during initial therapy (study 4). The mean \pm SEM of the admission plasma volumes of group 2 patients was $53.1 \pm 2.2 \mathrm{ml} / \mathrm{kg}$ body weight. An average of $79 \mathrm{~h}$ (range 66-96) later, the mean plasma volume (62.0 \pm 4.1 $\mathrm{ml} / \mathrm{kg})$ was significantly higher $(t=1.815, d f=10, P<0.05)$. The percentage plasma volume expansion $(17.3 \pm 7.8 \%)$ correlated significantly with the simultaneous fall in venous hematocrit $(24.0 \pm 4.4 \% ; r=0.64, P<0.05)$.

Validation of the model and its application to individual patients. From the three-component curve fitted to mean data, the population coefficient $A$ in $f_{1}(t)$ was $4.9 \%$. In terms of the model and from application of Eq. 9 above, this represents a mean initial expansion of the circulating plasma volume of

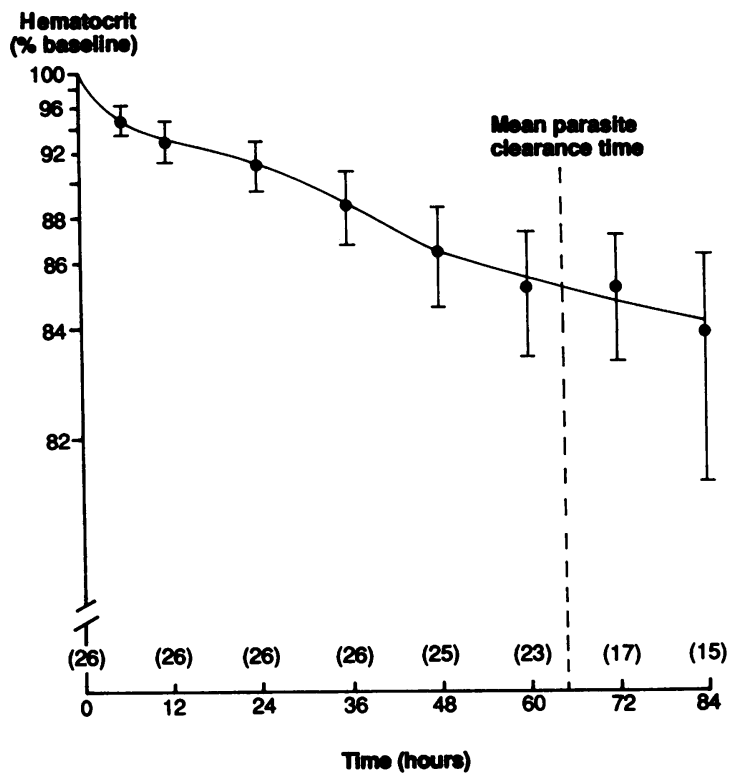

Figure 6. Semilogarithmic plot of geometric mean \pm SEM of parasite count expressed as a percentage of the admission value (baseline) for 17 patients with severe falciparum malaria. The solid line represents a zero-order regression line fitted to the data of the form $y=97.0$ $-2.792 x$. 
$7.5 \%$ relative to that on admission. The second component of the curve $\left[f_{2}(t)\right]$ reflects elimination of all parasitized cells equivalent to a further $6.2 \%$ fall in hematocrit. The average peripheral parasitemia of the 26 patients was $4.5 \%$. From Eq. 11 above and applying the population estimate of $s$ of 0.70 , the average volume of the sequestered biomass (exclusive of associated plasma) on admission was 1.06 times that of the peripheral parasite burden. The rate constant $c$ in $f_{3}(t)$ describes the exponential decline in hematocrit reflecting destruction of nonparasitized cells. In a manner analogous to that used to calculate mean cell lifespan from steady-state radiochromium data $(8)$, the mean cell lifespan of red cells derived from the half-time of $f_{3}(t)$ was $62 \pm 23 \mathrm{~d}$.

During the first $79 \mathrm{~h}$ of treatment, the model predicts that the plasma volume will increase through initial rehydration $(7.5 \%)$, replacement of the space vacated by destruction of both circulating parasitized cells (equivalent to $2.3 \%$ of $p$ ) and sequestered cells (equivalent to $2.4 \%$ of $p$ ), and through expansion to compensate for loss of nonparasitized cells (8.9\%). The total increase in volume in this group is, therefore, predicted to be $21.2 \%$ which is comparable to the mean $17.3 \%$ increase determined radioisotopically for group 2 patients over an average of $79 \mathrm{~h}$.

To assess the applicability of the model to individual cases, Eq. (12) above was fitted to data from 18 group 1 patients (mean admission venous hematocrit $0.37 \pm 0.05$ ) with at least seven valid hematocrit values over the first $84 \mathrm{~h}$ of treatment (see Table III). Parameter values were consistent with those derived from mean group 1 hematocrit data. The mean fall in venous hematocrit represented by $f_{1}(t)(3.9 \%)$ was equivalent to an average expansion in plasma volume of $7.2 \pm 3.3 \%$. The mean \pm SEM admission parasitemia of the 18 patients was $5.6 \pm 4.9 \%$. Estimates of the sequestered cell mass obtained from application of Eq. 11 and assuming a value of $s$ of 0.70 were $13.7 \pm 3.5 \%$. Two of five group 1 patients with cerebral malaria who were not transfused during the first $60 \mathrm{~h}$ of treatment had admission parasitemias of 0.5 and $0.1 \%$, and simultaneous model-derived sequestered cell volumes of $17.6 \%$ and $23.0 \%$, respectively. The ratio of circulating to sequestered cell volumes in these two patients were the greatest of the 18 individual values obtained.

\section{Discussion}

The model described in this report is an attempt to investigate the nature and extent of erythrocyte sequestration in severe falciparum malaria based on division of the intravascular volume into compartments representing circulating and sequestered red cells and plasma. Under the model's assumptions, serial changes in peripheral venous hematocrit provide an indirect estimate of the total sequestrum volume. Further analysis of the initial relationship between the two compartments yields an estimate of the hematocrit of sequestered cells and plasma $(s)$.

The model-derived average value of $s(0.70)$ was more than twice the mean admission venous hematocrit of group 1 patients $(0.33)$ and is consistent with semiquantitative electron microscopic descriptions of microvascular packing of sequestered erythrocytes (most of which contain mature parasites) in patients who died of cerebral malaria (16). Had there been significant pre-presentation red cell destruction but little se- questration, the value of $s$ would have been close to 1.0. The fact that $s$ is significantly less than unity is evidence that sequestered cells occupy a measurable volume within the circulation. However, this population estimate of $s$ gives no indication of variations between and within individuals.

Estimation of the sequestered cell volume from the model depends on the premise that no erythrocytes enter the circulation from either the sequestered biomass or bone marrow. Consistent with this and with the assumption that a $1.0 \% / \mathrm{d}$ elution rate can be validly applied to the labeled sample, the proportion of circulating radiochromium-labeled cells remained close to $100 \%$ of the admission value during the subsequent $84 \mathrm{~h}$. In addition, reticulocyte counts in a subset of patients did not rise during the first $4 \mathrm{~d}$ of illness. This feature of acute malaria has been reported previously $(3,17,18)$, particularly in severe infections.

A limitation of the model concerns the assumption that the whole body to peripheral venous hematocrit ratio is constant throughout initial treatment. The ratio is $\sim 0.90$ in normal individuals due to a low microvascular hematocrit $(19,20)$. Deviations from this figure are found in conditions such as chronic anemia and splenomegaly (13) and estimates of from 0.79 to 0.91 have been made in small numbers of patients with malaria (21). However, techniques for calculating whole body (circulating) hematocrit may not be valid during initial therapy because of a changing sequestrum volume, and factors such as an increase in spleen size could change the ratio during acute malaria. At present, there is no valid method for serial estimation of the magnitude of such changes.

Acute plasma volume expansion is likely to reflect rehydration in the presence of an increased vascular capacitance resulting from malaria (22) and antimalarial therapy (23). Evidence from studies of quinine disposition suggests that this expansion occurs within hours $(5,6)$. The first of three contemporaneous terms modeling serial changes in the venous hematocrit was a first-order fall consistent with a mean $7 \%$ plasma volume increase over an average half-time of $2 \mathrm{~h}$. This suggests that effective rehydration is completed well within 12 $h$ in most patients with severe malaria.

Subsequent plasma volume increases, over days rather than hours, have been considered an adaptive response to a steadily falling hematocrit (2). By vacating a "hidden" intravascular space, destruction of sequestered cells would also contribute to compensatory plasma expansion. The second of the contemporaneous terms fitted to serial hematocrit data was a zero-order decline (mean $6.3 \%$ of the admission hematocrit) over an average of $51 \mathrm{~h}$. This is likely to reflect expansion of the plasma volume in response to destruction of circulating and sequestered parasitized cells. Most adult patients with cerebral malaria regain consciousness within three days of hospital admission (24), consistent with the assumption that elimination of most sequestered cells occurs within this time. However, the exact processes underlying clearance of sequestered cells are unknown.

The model-derived average volume of sequestered red cells (4.8\% of the admission hematocrit) was of similar magnitude to the average peripheral parasitemia ( $4.5 \%)$. This volume may be an overestimate due to continued invasion and removal of circulating erythrocytes. Although patients received prompt schizonticidal doses of quinine (5-7), it is unlikely that schizogony was arrested abruptly. However, there is some evidence that infected cells are returned unparasitized to the circulation 
after passage through the spleen (25). These two processes, which would be difficult to incorporate in the model, counterbalance each other and are unlikely to make a significant net contribution to changes in hematocrit. Assessed from thick blood films, parasite clearance took a mean of $64 \mathrm{~h}$, longer than the durations of the zero order declines derived from thin blood smear examination (36 h) and the fitted hematocrittime curve $(51 \mathrm{~h})$. However, even if the parasitemia-time plot were sigmoid, removal of the small numbers of parasites found in its tail would contribute little to changes in hematocrit. A largely zero-order posttreatment decline in peripheral parasitemia has been reported previously $(26,27)$, with the parasitemia at 36 hours small in relation to the admission count.

Parasite multiplication during the growth phase of the infection and the propensity of the spleen to remove more mature circulating forms (2) suggest that peripheral parasitemia in early and uncomplicated infections is, in most cases, greater than the sequestered biomass. However, our model predicts that a typical patient with severe malaria harbors equivalent volumes of sequestered and circulating parasitized erythrocytes at presentation, and two group 1 cases, both with cerebral malaria, each had a model-derived sequestrum volume substantially greater than the simultaneous peripheral parasitemia. These observations provide further evidence that sequestration contributes to vital organ dysfunction and indicate that, in some patients, the peripheral parasitemia can be a marked underestimate of total parasite burden.

The third component of the hematocrit-time curve was a slower first order decline than that attributable to rehydration. In terms of the model, this represents destruction of circulating nonparasitized erythrocytes. The mean cell lifespan value derived from the mean hematocrit-time curve $(62 \mathrm{~d})$ was similar to the 57-d mean found using conventional radiochromium techniques in patients studied after parasite clearance (9). Further validation of the model, including the constancy of the venous to total body hematocrit ratio, was provided by plasma volume measurements. The increase in plasma volume over the first $79 \mathrm{~h}$ of treatment predicted by the model was close to that found using radioisotopic methods in group 2 patients. Other authors have found that increases of similar magnitude (28) occur from an already expanded plasma volume $(21,29$, 30) during the first few days of treatment.

The model provides as a framework on which serial changes in the venous hematocrit in severe falciparum malaria can be interpreted. A semilogarithmic plot of venous hematocrit against time should appear similar to that in Fig. 5 and, in conjunction with careful clinical evaluation, may be of use in assessing and planning management. For example, in an unconscious patient with anemia and moderate peripheral parasitemia, a rapid zero-order fall in hematocrit within the first 24 $h$ suggests a large sequestered biomass which may underlie other vital organ dysfunction and herald the need for blood transfusion.

Further studies are needed to validate the assumptions and predictions made by the model. In addition, its application to patients in specific subgroups (such as those with cerebral malaria, splenomegaly, and hyperparasitemia) warrants further evaluation. Falciparum malaria remains a major cause of morbidity and mortality in tropical countries. Quantitation of the infection in severe disease is a necessary prerequisite to both an understanding of the pathophysiological processes involved and improvements in management.

\section{Acknowledgments}

We are very grateful to the director, Dr Sanga Boonamrung, and staff of Paholpolpayuhasena Hospital, Kanchanaburi, Thailand for their cooperation during the study. We also thank Professors Danai Bunnag and Tranakchit Harinasuta for their support, and Kamolrat Silamut and Varunee Desakorn for valuable technical assistance.

The study is part of the Wellcome-Mahidol University-Oxford Tropical Medicine Research programme funded by the Wellcome Trust of Great Britain.

\section{References}

1. Marchiafava, E., and A. Bignami. 1894. On Summer-autumnal Fever. The New Sydenham Society, London. 103-119.

2. White, N. J. 1986. Malaria: pathophysiology. Clin. Trop. Med. Commun. Dis. 1:55-90.

3. Phillips, R. E., S. Looareesuwan, D. A. Warrell, S. H. Lee, J. Karbwang, M. J. Warrell, N. J. White, C. Swasdichai, and D. J. Weatherall. 1986. The importance of anemia in cerebral and uncomplicated falciparum malaria: role of complications, dyserythropoiesis and iron sequestration. Q. J. Med. 58:305-323.

4. World Health Organization Malaria Action Programme. 1986 Severe and complicated malaria. Trans. R. Soc. Trop. Med. Hyg. 80(Suppl.): $1-50$.

5. White, N. J., S. Looareesuwan, D. A. Warrell, M. J. Warrell, P. Chanthavanich, D. Bunnag, and T. Harinasuta. 1983. Quinine loading dose in cerebral malaria. Am. J. Trop. Med. 31:1-5.

6. Davis, T. M. E., N. J. White, S. Looareesuwan, K. Silamut, and D. A. Warrell. 1988. Quinine pharmacokinetics in cerebral malaria: predicted plasma concentrations after rapid intravenous loading using a two-compartmental model. Trans. R. Soc. Trop. Med. Hyg. 82:542547.

7. White, N. J., S. Looareesuwan, D. A. Warrell, M. J. Warrell, D. Bunnag, and T. Harinasuta. 1982. Quinine pharmacokinetics and toxicity in cerebral and uncomplicated malaria. Am. J. Med. 73:564-572.

8. I. C. S. H. Panel on Diagnostic Applications of Radioisotopes in Hematology. 1971. Recommended methods for radioisotope red cell survival studies. Br. J. Haematol. 21:241-250.

9. Looareesuwan, S., A. H. Merry, R. E. Phillips, R. Pleehachinda, Y. Wattanagoon, M. Ho, P. Charoenlarp, D. A. Warrell, and D. J. Weatherall. 1987. Reduced erythrocyte survival following clearance of malarial parasitemia in Thai patients. Br. J. Haematol. 67:473-478.

10. Dacie, J. V., and S. M. Lewis. 1975. Practical Hematology. Churchill Livingstone, London. 309-311.

11. Dagher, F. J., J. H. Lyons, and D. C. Finlayson. 1965. Blood volume measurement: a critical study prediction of normal values. Controlled measurement of sequential changes: choice of a bedside method. Adv. Surg. 1:69-109.

12. Bentley, S. A., H. S. Glass, S. M. Lewis, and L. Szur. 1974. Elution correction in ${ }^{51} \mathrm{Cr}$ red cell survival studies. $\mathrm{Br}$. J. Haematol. 22:179-192.

13. Davies, J. W. L. 1971. Blood volume studies. In Radioisotopes in Medical Diagnosis. E. H. Belcher and H. Vetter, editors. Butterworths, London. 319-341.

14. Armitage, P., and G. Berry. 1987. Statistical Methods in Medical Research. Blackwell Scientific Publications, Oxford, UK. 559 pp.

15. Metzler, C. M., and D. L. Weiner. 1984. PCNONLIN User's Guide. Statistical Consultants Inc., Lexington, MA. 68 pp.

16. McPherson, G. G., M. J. Warrell, N. J. White, S. Looareesuwan, and D. A. Warrell. 1985. Human cerebral malaria: a quantitative ultrastructural analysis of parasitized erythrocyte sequestration. Am. J. Pathol. 119:385-401.

17. Canfield, C. J. 1969. Renal and hematologic complications of acute falciparum malaria in Vietnam. Bull. NY Acad. Med. 45:10431057. 
18. Abdalla, S., D. J. Weatherall, S. N. Wickramasinghe, and M. Hughes. 1980. The anemia of $P$. falciparum malaria. Br. J. Haematol. 46:171-183.

19. Fahreus, R. 1929. The suspension stability of the blood. Physiol. Rev. 9:241-274.

20. Ebert, R. V., and E. A. Stead. 1941. Demonstration that the cell plasma ratio of blood contained in minute vessels is lower than that of venous blood. J. Clin. Invest. 20:317-321.

21. Malloy, J. P., M. H. Brooks, and K. G. Barry. 1967. Pathophysiology of acute malaria. II. Fluid compartmentalization. Am. J. Med. 43:745-750.

22. Brooks, M. H., J. P. Malloy, P. Bartelloni, W. D. Tigertt, T. W. Sheehy, and K. G. Barry. 1967. Pathophysiology of acute falciparum malaria: correlation of clinical and biochemical abnormalities. Am. J. Med. 43:735-744.

23. Kofi Ekue, J. M., D. E. Phiri, M. Mukunyandela, U. K. Sheth, and W. H. Wernsdorfer. 1988. Severe orthostatic hypotension during treatment of falciparum malaria. Br. Med. J. 296:396.

24. Warrell, D. A., S. Looareesuwan, M. J. Warrell, P. Kasemsarn, R. Intaraprasert, D. Bunnag, and T. Harinasuta. 1982. Dexametha- sone proves deleterious in cerebral malaria: a double blind trial in 100 comatose patients. $N$. Engl. J. Med. 306:313-319.

25. Schnitzler, B., T. Sodeman, M. L. Mead, and P. G. Contacos. 1972. Pitting function of the spleen in malaria: ultrastructural observation. Science (Wash. DC). 177:175-177.

26. Li, G., K. Arnold, X. Guo, H. Jian, and L. Fu. 1984. Randomised comparative study of mefloquine, qinghaosu, and pyramethamine-sulfadoxine in patients with falciparum malaria. Lancet. ii:1360-1361.

27. Jiang, J., G. Li, X. Guo, Y. Kong, and K. Arnold. 1982. Antimalarial activity of mefloquine and qinghaosu. Lancet. ii:285-288.

28. Chongsuphajaisiddhi, T., R. Kasemsuth, S. Tejavanija, and T. Harinasuta. 1971. Changes in blood volume in falciparum malaria. Southeast Asian J. Trop. Med. Public Health 2:344-350.

29. Feldman, H. A., and F. D. Murphy. 1945. The effect of alterations in blood volume on the anemia and hypoproteinemia of human malaria. J. Clin. Invest. 24:780-792.

30. Overman, R. R., T. S. Hill, and Y. T. Yong. 1949. Physiological studies in the human malarial host. I. Blood, plasma, 'extracellular' fluid volumes and ionic balance in therapeutic $P$. vivax and $P$. falciparum infections. J. Nat. Malar. Soc. 8:14-31. 